\title{
Sources, sinks and transport of energetic electrons near Saturn's main rings
}

E. Roussos ${ }^{1}$, P. Kollmann ${ }^{2}$, N. Krupp ${ }^{1}$, C. Paranicas ${ }^{2}$, K. Dialynas ${ }^{3}$, G.H.

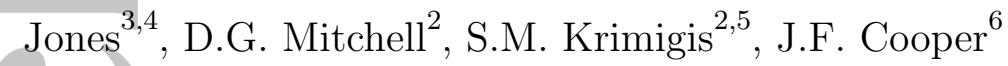

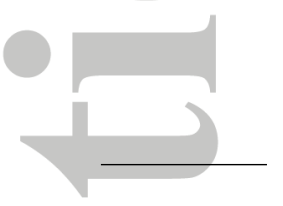

Elias Roussos, roussos@mps.mpg.de

${ }^{1}$ Max Planck Institute for Solar System

Research, 37077, Goettingen, Germany

${ }^{2}$ Johns Hopkins University Applied

Physics Laboratory, Laurel, MD

20723-6099, USA

${ }^{3}$ UCL Mullard Space Science Laboratory,

Dorking, Surrey RH5 6NT ,UK

${ }^{4}$ The Centre for Planetary Sciences at

UCL/Birkbeck, London WC1E 6BT, UK

${ }^{5}$ Office of Space Research and Technology

Academy of Athens, 11527, Greece

This article has been accepted for publication and undergone full peer review but has not been through the copyediting, typesetting, pagination and proofreading process, which may lead to differences between this version and the Version of Record. Please cite this article as doi: 10.1029/2018GL078097

(C)2018 American Geophysical Union. All Rights Reserved. 
The inner boundary of Saturn's electron radiation belts, near the planet's A-ring $\left(\sim 2.27 \mathrm{R}_{\mathrm{s}}\right)$, is studied using Cassini's Proximal orbit measurements. We find that variable convective flows transport energetic electrons to the A-ring, which absorbs them instantaneously, forming the inner belt boundary. These flows are also responsible for a variable and longitudinally asymmetric boundary configuration. Pre-noon, the boundary oscillates towards and away from the A-ring with a two-week period. Post-noon, it maps persistently near the F-ring $\left(\sim 2.32 \mathrm{R}_{\mathrm{s}}\right)$ and coexists with localized MeV electron intensity enhancements (microbelts). We propose that the microbelts contain electrons in drift resonance with corotation, trapped in local-time confined trajectories which result from the aforementioned convective flows. The microbelts' collocation with the F-ring implies either a local, secondary electron production due to Galactic Cosmic Ray collisions with F-ring dust, or an enhanced resonant electron trapping due to an electrodynamic interaction between the F-ring and Saturn's magnetosphere.

\section{Keypoints:}

- The radial transport of energetic electrons onto Saturn's rings is dominated by convection

${ }^{6}$ NASA Goddard Space Flight Center,

Greenbelt, Maryland, 11527, Greece

(C)2018 American Geophysical Union. All Rights Reserved. 
- The F ring contains regions of $\mathrm{MeV}$ electron pileup evolving separately from Saturn's electron belts

- The F-ring may be both a source and a sink of energetic electrons
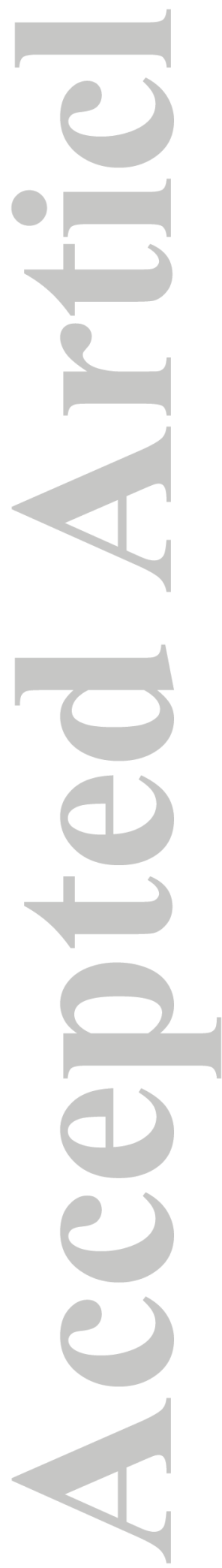

(C)2018 American Geophysical Union. All Rights Reserved. 


\section{Introduction}

It is generally assumed that the innermost distance where energetic charged particles can be trapped beyond Saturn's main rings maps to the L-shell of the A-ring's outer edge, at $\mathrm{L} \sim 2.27$ (the $\mathrm{L}$-shell, $L$, is normalized to a planetary radius, $1 \mathrm{R}_{\mathrm{S}}=60268 \mathrm{~km}$ ). That is because the exterior A-ring, with a surface mass density of $23.8 \pm 10.8 \mathrm{gcm}^{-2}[$ Spilker et al., 2004], contains enough material to absorb 30-100 MeV electrons and 200-350 MeV protons after few traversals through its surface. Indeed, measurements obtained during the Pioneer 11 flyby of Saturn [Simpson et al., 1980; Van Allen et al., 1980] and by Cassini [Paranicas et al., 2010] show fluxes of energetic protons and electrons dropping close to their background levels when the two spacecraft crossed onto field lines mapping to the A-ring. Still, the transition between the radiation belts and the main rings is more than just a region of a non-trivial energetic particle depletion: it is actually a site holding key information for understanding the belts' structure and dynamics.

For instance, the process dominating the transport of trapped particles onto the A-ring, which ultimately determines their loss time scales, appears to be different for energetic protons and electrons. The well-defined structure and stability of the proton radiation belts [Kollmann et al., 2017] indicate that the transport of protons is driven by slow, radial diffusion. Contrary to that, electron transport appears to be affected by global scale, cold plasma radial flows (alternately referred here as convection) because it has been observed that energetic electron transients propagate rapidly from the middle magnetosphere into the radiation belts, where they persist for less than a week. In order to explain the difference between protons and electrons, Roussos et al. [2018a] inferred that the electron

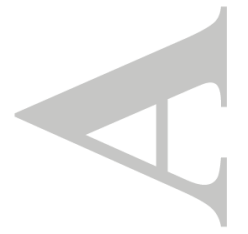

(C)2018 American Geophysical Union. All Rights Reserved. 
radiation belts contain a significant population of $\mathrm{MeV}$ electrons in near drift-resonance with corotation (meaning that their azimuthal gradient and curvature drift at an energy of $\mathrm{E}=\mathrm{E}_{c}$ cancels out corotation) [Thomsen and Van Allen, 1980]. Such electrons are particularly sensitive to convection, and they can be transported throughout the magnetosphere and to the rings in a relatively short time.

The only published study supporting a case of a fast, convective transport of electrons onto the rings with in-situ measurements from their vicinity is that of Paranicas et al. [2010], who found that the ring absorption of MeV electrons, observed by Cassini on day $183 / 2004$, was asymmetric in local time and displaced outwards with respect to $L \sim 2.27$ at noon. That single observation, however, could not rule out that Saturn's F-ring has also a role in creating such asymmetries. The F-ring is a longitudinally asymmetric and dynamic ring located just outside the main rings, between $\sim 2.30$ and $\sim 2.34 \mathrm{R}_{\mathrm{S}}$. Besides its 10-50 km wide dusty core, that comprises 10-1000 $\mu \mathrm{m}$ ice grains and has a mean optical depth $(\tau)$ of $\sim 0.2$ [Showalter et al., 1992; Bosh et al., 2002; Becker et al., 2018], the F-ring contains moonlets in the size range of $0.1-1 \mathrm{~km}$ and dust clumps with arc lengths typically exceeding $5^{\circ}$ of longitude [Esposito et al., 2008; French et al., 2014]. These moonlets and clumps appear to be efficient sinks of energetic electrons [Van Allen, 1982; Cuzzi and Burns, 1988].

During Cassini's Proximal orbits (days 113-258/2017) the L-shells of the F-ring and the A-ring edge were crossed 45 times, providing a unique opportunity to study those rings' interactions with the electron belts in a systematic way. An initial, brief description of these observations is provided in Buratti et al. [2018]. Besides identifying several ring and

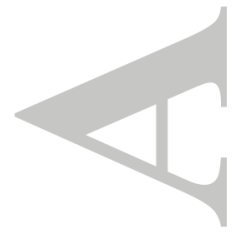

(C)2018 American Geophysical Union. All Rights Reserved. 
moon driven losses of energetic protons and electrons, those authors reported the detection of a series of unexpected, localized enhancements of energetic electron intensities in the vicinity of the A and F rings, which here we refer to as "microbelts". Their occurrence appears to be asymmetric in local time so their study could be central for understanding convective flows near the rings.

In the present study we expand on the initial observations by Buratti et al. [2018] and touch upon several of the open questions summarized above, the answers to which we believe are interlinked: (a) is the transport of energetic electrons on the rings diffusive or convective, (b) is the F-ring an important sink of energetic electrons and (c) what is the origin of the microbelts? Important contextual information is also provided by energetic electron measurements above Saturn's main rings.

\section{Dataset information}

We analyze energetic electron measurements from Cassini’s Low Energy Magnetospheric Measurement System (LEMMS), which is part of the Magnetosphere Imaging Instrument (MIMI) [Krimigis et al., 2004]. LEMMS is a particle telescope providing information on the particles' energy, species and direction above about $20 \mathrm{keV}$ and well into the $\mathrm{MeV}$ range. Here we analyze data from LEMMS's rate and priority channels that monitor MeV electrons. The priority channels accumulate counts every $\sim 0.3 \mathrm{~s}, 16$ times faster than the rate channels with the same energy response and are therefore useful for resolving the microbelts.

All measurements were obtained during Cassini's Proximal orbits. Each full orbit included two crossings of the ring-belt transition at a latitude of $\sim 43^{\circ}$ north (inbound) or

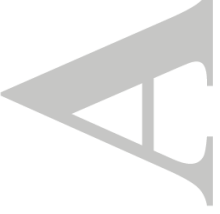

(C)2018 American Geophysical Union. All Rights Reserved. 
south (outbound). One more crossing took place during the final partial orbit, about an hour before the end of mission (day 258/2017 10:31:45 UTC). The local time of the inbound crossings was between 9:10 and 10:50 and between 13:10 and 14:50 outbound. Cassini's position has been mapped to the equatorial plane using an empirical third-order multipole model for Saturn's internal magnetic field as in Cao et al. [2011] (see supplement). The equatorial pitch angle coverage of LEMMS was narrow $\left(10^{\circ}-22^{\circ}\right)$.

\section{Observations}

Figure 1 displays the L-shell profile of energetic particle count rates from three orbits that are representative of all the measurements obtained at the L-shells of the $\mathrm{A}$ and $\mathrm{F}$ rings during the Proximal orbits. Rates of $>12 \mathrm{MeV}$ protons (red) and $>800 \mathrm{keV}$ electrons

(black) are uncorrelated, meaning that electron measurements are not contaminated by protons.

Several features stand out: in all inbound crossings, the electron rates reach the background levels at $\mathrm{L} \sim 2.34$, close to the outer edge of the F-ring and no microbelts are visible. The outbound crossings show the microbelts within the F-ring boundaries. Some residual foreground electron signal is also seen between the A and $\mathrm{F}$ rings $(2.27<L<2.30)$. These inbound-outbound differences are indicative of a highly asymmetric configuration of the ring-belts transition within just few hours of local time.

We extracted manually the coordinates of the microbelts' location and peak count rate and those of the inner boundary of the electron radiation belts. The latter is defined as the minimum L-shell $\left(L_{b}\right)$ where the $>800 \mathrm{keV}$ electron priority rates drop below 4 counts $^{-1}$. Choosing a lower threshold down to 3 counts $^{-1}$ ) had a negligible impact

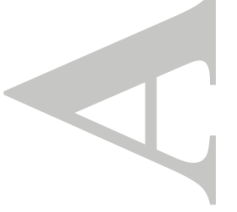

(C)2018 American Geophysical Union. All Rights Reserved. 
on the results that we present below. Even lower thresholds were avoided to prevent confusing a boundary crossing with random signal fluctuations. For that purpose, we also averaged the priority rates over $2 \mathrm{~s}$.

The values of $L_{b}$ are plotted in Figure 2A (red diamonds). The mean L-shell of the inner boundary is $\left\langle L_{b}\right\rangle=2.32 \pm 0.02$, effectively contained within the F-ring boundaries. Between 9:10 and 10:50 hours $L_{b}$ has a larger scatter $\left(\left\langle L_{b(\text { inb. })}\right\rangle=2.33 \pm 0.03\right)$ than its "global" average. This scatter appears to be driven by temporal effects: a strong, two week periodicity is seen in the time-series of $L_{b}$ (Figure 2B, red profile), possibly driven by the interaction of Corotating Interaction Regions (CIRs) with Saturn's magnetosphere, as we discuss in Section 4.1. In the post-noon local time sector $L_{b}$ is better constrained within the F-ring with $\left\langle L_{b(\text { outb. })}\right\rangle=2.31 \pm 0.02$ (Figure $\left.2 \mathrm{~A}\right)$.

Interestingly, no periodicity is seen in $L_{b}$ post-noon (Figure 2B, black profile). That may be due to the fact that $L_{b}$ in this local time sector is determined by the presence of the microbelts, the L-shell of which $\left(L_{s}\right)$ is plotted with gray diamonds (Figure 2A) and was always within $\delta L<0.02$ outside $L_{b}$.

Out of the 22 microbelts observed, 18 were located post-noon and 4 pre-noon. Their mean L-shell is $\left\langle L_{s}\right\rangle=2.33 \pm 0.01$, while their L-shell width, shown as an error bar in Figure $2 \mathrm{~A}$, is $\langle W\rangle=0.02 \pm 0.01$. The microbelts are clearly mapped within the F-ring boundaries.

\section{Discussion}

Even though only a limited local time range was sampled during Cassini's Proximal Orbits, all observations summarized above indicate that the ring-belt interface is very

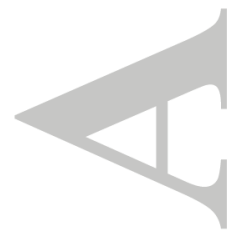

(C)2018 American Geophysical Union. All Rights Reserved. 
dynamic and asymmetrically structured. We now discuss the implications of several of these observations.

\subsection{The origin of the inner electron radiation belt boundary}

All but four of the inner electron radiation belt boundaries were at $L_{b}>2.30$. Two are the possible explanations for this observation.

The first is that the F-ring is an important energetic electron sink. For an F-ring core with $\tau \sim 0.2$ and an effective dust particle size lower than $50 \mu \mathrm{m}$ ) [Bosh et al., 2002], the surface mass density is below $10^{-3} \mathrm{~g} \mathrm{~cm}^{-2}$, a value sufficient for stopping $25 \mathrm{keV}$ electrons or gradually moving $\mathrm{MeV}$ electrons below the $800 \mathrm{keV}$ energy limit of the LEMMS channel used here. Moonlets, dust clumps or longitudinal F-ring sectors with higher dust density would enhance the global losses of electrons.

The second possibility, which we favour, is that electron absorption happens at the A-ring (e.g. around midnight) but its effects are propagated to larger L-shells due to convection. The colocation of the inner belt boundary with the F-ring may then be a misleading coincidence. The outward offset of $L_{b}$ could be driven by a uniform electric field pointing in the general direction of midnight, as we observe at larger L-shells $(\mathrm{L}>4)$ [Andriopoulou et al., 2012; Thomsen et al., 2012; Wilson et al., 2013]. This electric field, typically referred to as "noon-to-midnight electric field", causes a uniform, dawnward $\mathrm{E} \times \mathrm{B}$ convection of particles, that shifts their otherwise circular orbits in the direction of noon $\left(\mathrm{E}<\mathrm{E}_{c}\right)$ or midnight $\left(\mathrm{E}>\mathrm{E}_{c}\right)$. The magnitude of the offset maximizes near $\mathrm{E}=\mathrm{E}_{c}$, although at $\mathrm{E}_{c}$ drift orbits become more complex (Section 4.2.1). If the model of A-ring

electron absorption at midnight is valid, then $\mathrm{E}<\mathrm{E}_{c}$ and the intensity of the electric field

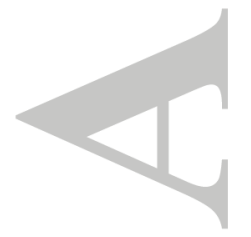

(C)2018 American Geophysical Union. All Rights Reserved. 
needed to account for the observed dayside, outward offset of the resulting depletion is $<0.5 \mathrm{mV} \mathrm{m}^{-1}$ [Paranicas et al., 2010].

The reason we favour the second scenario is because in the pre-noon sector $L_{b}$ was observed outside the F-ring $(2.34<\mathrm{L}<2.37)$ for $50 \%$ of the orbits. The two-week periodicity identified in the pre-noon sector's $L_{b}$ values (Figure $2 \mathrm{~B}$ ) hints that the strength of convection is modulated by the interaction of Saturn's magnetosphere with the solar wind. A similar periodicity has been observed in the intensity and extent of the electron radiation belts in 2008 [Roussos et al., 2014] and was attributed to Saturn's magnetosphere interaction with two CIRs every solar rotation [Roussos et al., 2018b]. Evidence that Saturn was almost continuously exposed to CIRs during the Proximal Orbits is provided in Roussos et al. [2018c]. By comparison, convection in Jupiter's inner magnetosphere is also regulated by solar wind transients Murakami et al. [2016].

A question that then emerges is why post-noon we do not observe a similar temporal modulation, as these electrons would be exposed to the same global convective flows. Since the value of $L_{b}$ post noon is controlled by the microbelts, we should consider whether the microbelts' electron source is not the same as for the radiation belt electrons observed pre-noon. Before exploring this possibility, we should first understand how the microbelts can be so localized.

\subsection{The origin of the microbelts}

\subsubsection{Local time confinement}

A pile-up of electron flux restricted in local time may occur due to the presence of corotation-resonant electrons in the electron distribution $\left(\mathrm{E} \sim \mathrm{E}_{c}\right)$. Global convective flows

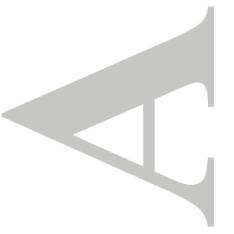

(C)2018 American Geophysical Union. All Rights Reserved. 
would force such electrons to follow closed paths in a narrow local time range, rather than the typical, circumplanetary trajectories [Cooper et al., 1998].

Such an example is shown in Figure 3A, where we plot the bounce-averaged, guiding center trajectories of 36 resonant electrons injected uniformly in local time at $\mathrm{L}=2.32$. The electrons were traced with the method of Roussos et al. [2013] under the influence of corotation, dipole magnetic drifts and a noon to midnight electric field of $0.025 \mathrm{mV} \mathrm{m}^{-1}$.

The closed trajectories of electrons centered around noon, opposite to the electric field's pointing, are clearly visible.

Only electrons injected between 10:00 and 13:00 complete their non-circumplanetary, closed paths. If there is a continuous supply of electrons in that region (e.g. through a local source or transient periods of enhanced convection [Roussos et al., 2018a]), electron fluxes could build up, forming localized maxima, i.e. the microbelts. A stronger convective electric field increases the L-shell range and narrows down the local time extent of these closed paths. Since observations indicate that microbelts span at least two hours of local time and their L-shell width, $W$, is lower than 0.03 , we can estimate that the electric field strength cannot be much stronger than $0.1 \mathrm{mV} \mathrm{m}^{-1}$.

When such electrons are injected at local times other than 10:00-13:00, they drift onto the A-ring where they get absorbed between 13:00 and 22:00 forming forbidden access (F.A.) regions in the pre-noon sector. F.A. regions at pre-noon local times are seen also for electrons with energies slightly below or above the corotation resonant energy (Figure $3 \mathrm{~B}, \mathrm{C})$ that intersect the A-ring. That could explain the lack of electrons inward of $\mathrm{L} \sim 2.29$ and between 9:00 and 11:00 (Figure 2A).

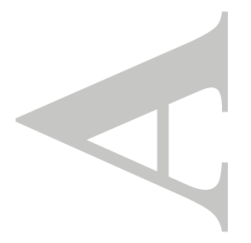

(C)2018 American Geophysical Union. All Rights Reserved. 
For energies which electron orbits do not intersect the A-ring (Figures 3D, E), the F.A. regions become symmetric around the noon-midnight direction and smaller and thus contribute less to the asymmetries seen with LEMMS. Finally, since the sinusoidal amplitude and phase of the orbits changes strongly with energy (Figure 3F), most electrons that contribute to the broad $>800 \mathrm{keV}$ LEMMS channel would disperse over a wide Lshell and local time range in the presence of convection. Essentially, unless $\mathrm{E} \sim \mathrm{E}_{c}$, local time confinement of electrons is not favored.

A change in the electric field pointing from the noon-midnight direction, shifts accordingly the location of the confined electron trajectories and the F.A. regions discussed above. If indeed the local time of the microbelts indicates the electric field's direction, we estimate that its most probable pointing is after 01:00 hours. No constrain can be placed for the pointing towards the dawn direction due to the limited local time coverage of the Proximal orbits. This direction agrees with the post-midnight pointing of the global convective electric field found by Wilson et al. [2013] and Andriopoulou et al. [2013].

\subsubsection{L-shell confinement and F-ring localization}

The local time confinement described above can occur at any L-shell where corotation resonant electrons exist. Since beyond the dense A-ring there is no strong absorber to limit their pile-up, then why are microbelts restricted at $\mathrm{L}<2.35$ and centered at the L-shell of the F-ring?

One possibility is that an electrodynamic interaction of the F-ring with the magnetosphere drives local plasma flow perturbations (e.g. due to mass loading) which could lead to a preferential trapping of energetic electrons at those ring's location. Such electrons

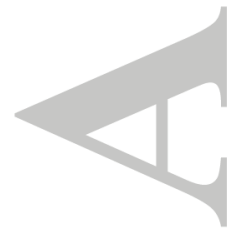

(C)2018 American Geophysical Union. All Rights Reserved. 
could come directly from Saturns radiation belts, which may be dominated by electrons around $\mathrm{E}=\mathrm{E}_{c}$ [Roussos et al., 2018a]). A caveat of this scenario is that it does not explain why microbelts are organized with local time: a ring-magnetosphere interaction should take place at all local times. Even though we do not favor the aforementioned concept, we cannot exclude it as we have no clear understanding at this stage on how the interaction between the magnetosphere with a highly structured F-ring would look like.

A second scenario involves the production of energetic electrons from the F-ring due to its irradiation by Galactic Cosmic Rays (GCRs). Such an interaction may release electrons in two ways. The first is through the Cosmic Ray Albedo Neutron Decay (CRAND) [Blake et al., 1983], which supplies electrons over a wide L-shell range and cannot account for the L-shell localization of the microbelts. The second is through a direct electron release from the decay of pions which get produced in GCR-ring collisions: $\pi^{ \pm} \rightarrow \mu^{ \pm} \rightarrow e^{ \pm}[$Chenette et al., 1980].

This process generates electrons locally and over a wide energy range [Cooper et al., 1985] that includes $\mathrm{E}_{c}$. Since the F-ring moonlets and clumps may carry significant column mass density that compares to that of the A-ring, we analyzed LEMMS observations from the times that Cassini was magnetically connected to the main rings in order to understand if and how many secondary electrons get produced by GCRs. Any foreground electron signal above the main rings may only come from GCR secondaries.

Figure 4 shows Proximal orbit averaged rates of $>800 \mathrm{keV}$ electrons as a function of L-shell across the main rings. This signal, that was observed with much lower spatial resolution by Pioneer 11 [Chenette et al., 1980], has been attributed to secondary electrons

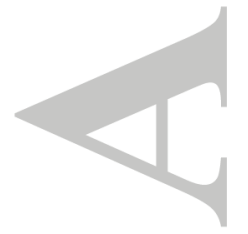

(C)2018 American Geophysical Union. All Rights Reserved. 
generated from the impact of GCRs on the atmosphere and/or the rings. The drop of the signal towards the planet is due to the reduced GCR access with decreasing L-shell [Kotova, 2016], which translates into a lower production of secondaries. Any superimposed deviation from this trend signifies a local change in the density of the material that GCRs interact with. The observation of a region with reduced electron production at the expected position of the Cassini division, proves that these electrons come from the rings and not the atmosphere.

The average, background subtracted count-rate of ring electrons produced at the outer A-ring is $\sim 0.9$ counts $^{-1}$. Since these electrons will have a maximum lifetime of half a bounce period $(\sim 1 \mathrm{~s}), 0.9$ counts $^{-1}$ could also correspond to the approximate increase the $>800 \mathrm{keV}$ electron signal every second at a location of the F-ring where production is similarly effective. In the absence of losses and even if just $1 \%$ of the secondary electrons have energies near the corotation resonance, the signal observed in the microbelts $(\sim 50$ 100 counts $^{-1}$ ) could accumulate within 1.5-3 hours, while corotation resonant electrons complete their trajectories over one week.

What limits the signal intensity in the microbelts is that following their production, the secondary electrons are immediately exposed to the F-ring. It is possible, however, that the production is stronger than the loss rate because electrons may drift relative to the localized F-ring sectors that produced them (which orbit Saturn with the keplerian velocity) and escape regions of high column mass density within seconds or minutes of their release into the radiation belts.

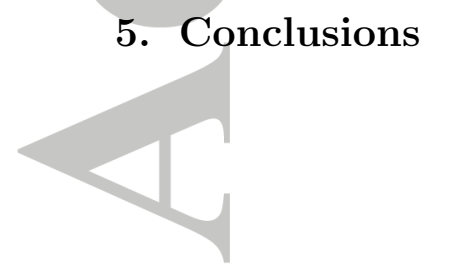

(C)2018 American Geophysical Union. All Rights Reserved. 
We discussed several concepts in order to explain the topological characteristics of the inner electron belt boundary $\left(L_{b}\right)$ and of the microbelts. All explanations had a common requirement: convective flows should be present near Saturn's rings meaning that the transport of energetic electrons onto the rings is convective and not diffusive. In that respect, our results on whether whether electron losses on the F-ring contribute to the formation of $L_{b}$ are less clear.

We have shown evidence that the strength of convection may be modulated by CIRs, revealing the influence of the solar wind very deep into Saturn's radiation belts. That suggests that a significant component of the electric field modulating $L_{b}$ has its origin in the magnetosphere. It may be relevant to the noon-midnight electric field identified at larger L-shells [Andriopoulou et al., 2013; Thomsen et al., 2012; Wilson et al., 2013].

A caveat of this connection is that these previous studies predicted electric field strengths $>0.1 \mathrm{mV} \mathrm{m}^{-1}$ near the rings, higher than estimated here. Besides that, uniform convection could not explain similarities in the asymmetries of $\sim 1 \mathrm{MeV}$ and $>7 \mathrm{MeV}$ electrons near the rings seen by Cassini in 2004 [Paranicas et al., 2010; Thomsen et al., 2012]. Bridging these inconsistencies may require us to move past the simplified models of steady-state convection and the uniform electric field to describe non-corotational flows used in the current and past studies, especially in light of the CIR-modulated $L_{b}$ value shown here and the findings of Roussos et al. [2018a].

Another implication of our analysis is that the F-ring may be a direct source of energetic electrons in the magnetosphere, through its interaction with GCRs. An F-ring source is required to explain the origin of the microbelts within those ring's boundaries and justify

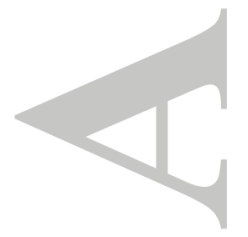

(C)2018 American Geophysical Union. All Rights Reserved. 
why $L_{b}$ at post-noon local times does not show the same periodic modulation as observed pre-noon. An alternative scenario for the formation of the microbelts involves trapping of electrons in regions that there may be a strong electrodynamic interaction between the F-ring and the magnetosphere. Simulations, the analysis of lower energy electron data of LEMMS and context from other instruments would be required to decide if any of these two concepts is relevant.

\section{Acknowledgments.}

We thank Andreas Lagg (MPS), Jon Vandegriff and Martha Kusterer (JHUAPL) for software support and data reduction. All raw MIMI data shown here will be will be released via NASA's Planetary Data System within 2018.

\section{References}

Andriopoulou, M., E. Roussos, N. Krupp, C. Paranicas, M. Thomsen, S. Krimigis, M. K. Dougherty, and K.-H. Glassmeier (2012), A noon-to-midnight electric field and nightside dynamics in Saturn's inner magnetosphere, using microsignature observations, Icarus, 220, 503-513, doi:10.1016/j.icarus.2012.05.010.

Andriopoulou, M., E. Roussos, N. Krupp, C. Paranicas, M. Thomsen, S. Krimigis, M. Dougherty, and K.-H. Glassmeier (2013), Spatial and temporal dependence of the convective electric field in saturns inner magnetosphere, Icarus, doi: http://dx.doi.org/10.1016/j.icarus.2013.10.028.

Becker, T. M., J. E. Colwell, L. W. Esposito, N. O. Attree, and C. D. Murray (2018), Cassini uvis solar occultations by saturns f ring and the detection of collision-produced

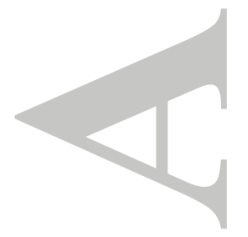

(C)2018 American Geophysical Union. All Rights Reserved. 
micron-sized dust, Icarus, doi:10.1016/j.icarus.2018.02.006.

Blake, J. B., H. H. Hilton, and S. H. Margolis (1983), On the injection of cosmic ray secondaries into the inner Saturnian magnetosphere. I - Protons from the CRAND process, Journal Geophys. Res., 88, 803-807, doi:10.1029/JA088iA02p00803.

Bosh, A. S., C. B. Olkin, R. G. French, and P. D. Nicholson (2002), Saturn's F Ring: Kinematics and Particle Sizes from Stellar Occultation Studies, Icarus, 157, 57-75, doi:10.1006/icar.2002.6791.

Buratti, B., et al. (2018), First results from cassini's five fabulous flybys of saturn's ring moons, Science.

Cao, H., C. T. Russell, U. R. Christensen, M. K. Dougherty, and M. E. Burton (2011), Saturn's very axisymmetric magnetic field: No detectable secular variation or tilt, Earth and Planetary Science Letters, 304(1), 22 - 28, doi: https://doi.org/10.1016/j.epsl.2011.02.035.

Chenette, D. L., J. F. Cooper, J. H. Eraker, K. R. Pyle, and J. A. Simpson (1980), High-energy trapped radiation penetrating the rings of saturn, J. Geophys. Res. (Space Physics), 85(A11), 5785-5792, doi:10.1029/JA085iA11p05785.

Cooper, J. F., J. H. Eraker, and J. A. Simpson (1985), The secondary radiation under Saturn's A-B-C rings produced by cosmic ray interactions, Journal Geophys. Res., 90, 3415-3427, doi:10.1029/JA090iA04p03415.

Cooper, J. F., E. C. Sittler, S. Maurice, B. H. Mauk, and R. S. Selesnick (1998), Local time asymmetry of drift shells for energetic electrons in the middle magnetosphere of saturn, Advances in Space Research, 21, 1479-1482, doi:10.1016/S0273-1177(98)00022-2.

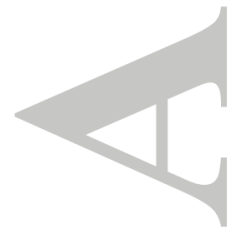

(C)2018 American Geophysical Union. All Rights Reserved. 
Cuzzi, J. N., and J. A. Burns (1988), Charged particle depletion surrounding Saturn's F ring - Evidence for a moonlet belt?, Icarus, 74, 284-324, doi:10.1016/0019$1035(88) 90043-7$.

Esposito, L. W., B. K. Meinke, J. E. Colwell, P. D. Nicholson, and M. M. Hedman (2008), Moonlets and clumps in saturn's f ring, Icarus, 194(1), 278 - 289, doi: https://doi.org/10.1016/j.icarus.2007.10.001.

French, R. S., S. K. Hicks, M. R. Showalter, A. K. Antonsen, and D. R. Packard (2014), Analysis of clumps in saturns f ring from voyager and cassini, Icarus, 241, $200-220$, doi:https://doi.org/10.1016/j.icarus.2014.06.035.

Kollmann, P., E. Roussos, A. Kotova, C. Paranicas, and N. Krupp (2017), The evolution of Saturn's radiation belts modulated by changes in radial diffusion, Nature Astronomy, doi:10.1038/s41550-017-0287-x.

Kotova, A. (2016), Energetic particle tracking techniques and its application to the magnetosphere of Saturn, Theses, Uninersite Toulouse III - Paul Sabatier.

Krimigis, S. M., D. G. Mitchell, D. C. Hamilton, S. Livi, J. Dandouras, S. Jaskulek, T. P. Armstrong, J. D. Boldt, A. F. Cheng, G. Gloeckler, J. R. Hayes, K. C. Hsieh, W.-H. Ip, E. P. Keath, E. Kirsch, N. Krupp, L. J. Lanzerotti, R. Lundgren, B. H. Mauk, R. W. McEntire, E. C. Roelof, C. E. Schlemm, B. E. Tossman, B. Wilken, and D. J. Williams (2004), Magnetosphere Imaging Instrument (MIMI) on the Cassini Mission to Saturn/Titan, Space Science Reviews, 114, 233-329, doi:10.1007/s11214-004-1410-8.

Murakami, G., K. Yoshioka, A. Yamazaki, F. Tsuchiya, T. Kimura, C. Tao, H. Kita, M. Kagitani, T. Sakanoi, K. Uemizu, Y. Kasaba, I. Yoshikawa, and M. Fujimoto (2016),

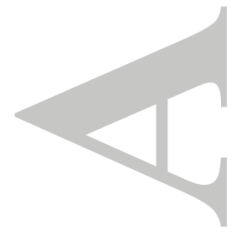

(C)2018 American Geophysical Union. All Rights Reserved. 
Response of jupiter's inner magnetosphere to the solar wind derived from extreme ultraviolet monitoring of the io plasma torus, Geophys. Res. Lett., 43(24), 12,308-12,316, doi:10.1002/2016GL071675, 2016GL071675.

Paranicas, C., D. G. Mitchell, S. M. Krimigis, J. F. Carbary, P. C. Brandt, F. S. Turner,

E. Roussos, N. Krupp, M. G. Kivelson, K. K. Khurana, J. F. Cooper, T. P. Armstrong, and M. Burton (2010), Asymmetries in Saturn's radiation belts, J. Geophys. Res. (Space Physics), 115(A14), A07216, doi:10.1029/2009JA014971.

Roussos, E., M. Andriopoulou, N. Krupp, A. Kotova, C. Paranicas, S. M. Krimigis, and D. G. Mitchell (2013), Numerical simulation of energetic electron microsignature drifts at Saturn: Methods and applications, Icarus, 226, 1595-1611, doi: 10.1016/j.icarus.2013.08.023.

Roussos, E., N. Krupp, C. Paranicas, J. F. Carbary, P. Kollmann, S. M. Krimigis, and D. G. Mitchell (2014), The variable extension of Saturn's electron radiation belts, Planetary Space Sci., 104, 3-17, doi:10.1016/j.pss.2014.03.021.

Roussos, E., P. Kollmann, N. Krupp, C. Paranicas, K. Dialynas, N. Sergis, D. Mitchell, D. Hamilton, and S. Krimigis (2018a), Drift-resonant, relativistic electron acceleration at the outer planets: Insights from the response of saturns radiation belts to magnetospheric storms, Icarus, 305, 160 - 173, doi:https://doi.org/10.1016/j.icarus.2018.01.016.

Roussos, E., C. Jackman, M. Thomsen, W. Kurth, S. Badman, C. Paranicas, P. Kollmânn, N. Krupp, R. Buk, D. Mitchell, S. Krimigis, D. Hamilton, and A. Radioti (2018b), Solar energetic particles (sep) and galactic cosmic rays (gcr) as tracers of solar wind conditions near saturn: Event lists and applications, Icarus, 300, 47 - 71,

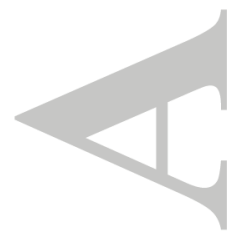

(C)2018 American Geophysical Union. All Rights Reserved. 
doi:https://doi.org/10.1016/j.icarus.2017.08.040.

Roussos, E., N. Krupp, C. Paranicas, P. K. D. Mitchell, S. Krimigis, B. Palmaerts, K. Dialynas, and C. M. Jackman (2018c), Heliospheric conditions during Cassini's ring grazing and proximal orbits, Geophys. Res. Lett., doi:10.1029/2018GL078093.

Showalter, M. R., J. B. Pollack, M. E. Ockert, L. R. Doyle, and J. B. Dalton (1992), A photometric study of Saturn's F Ring, Icarus, 100, 394-411, doi:10.1016/00191035(92)90107-I.

Simpson, J. A., T. S. Bastian, D. L. Chenette, G. A. Lentz, R. B. McKibben, K. R. Pyle, and A. J. Tuzzolino (1980), Saturnian trapped radiation and its absorption by satellites and rings - The first results from Pioneer 11, Science, 20\%, 411-415, doi: 10.1126/science.207.4429.411.

Spilker, L. J., S. Pilorz, A. L. Lane, R. M. Nelson, B. Pollard, and C. T. Russell (2004), Saturn a ring surface mass densities from spiral density wave dispersion behavior, Icarus, 171 (2), 372 - 390, doi:https://doi.org/10.1016/j.icarus.2004.05.016.

Thomsen, M. F., and J. A. Van Allen (1980), Motion of trapped electrons and protons in Saturn's inner magnetosphere, Journal Geophys. Res., 85, 5831-5834, doi: 10.1029/JA085iA11p05831.

Thomsen, M. F., E. Roussos, M. Andriopoulou, P. Kollmann, C. S. Arridge, C. P. Paranicas, D. A. Gurnett, R. L. Powell, R. L. Tokar, and D. T. Young (2012), Saturn's inner magnetospheric convection pattern: Further evidence, J. Geophys. Res. (Space Physics), 117, A09208, doi:10.1029/2011JA017482.

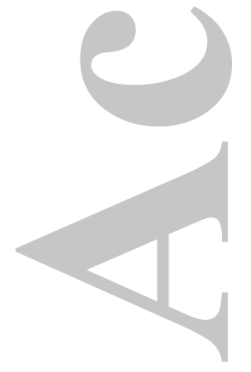

(C)2018 American Geophysical Union. All Rights Reserved. 
Van Allen, J. A. (1982), Findings on rings and inner satellites of Saturn of Pioneer 11, Icarus, 51, 509-527, doi:10.1016/0019-1035(82)90143-9.

Van Allen, J. A., M. F. Thomsen, B. A. Randall, R. L. Rairden, and C. L. Grosskreutz (1980), Saturn's magnetosphere, rings, and inner satellites, Science, 20\%, 415-421, doi: 10.1126/science.207.4429.415.

Wilson, R. J., F. Bagenal, P. A. Delamere, M. Desroche, B. L. Fleshman, and V. Dols (2013), Evidence from radial velocity measurements of a global electric field in saturn's inner magnetosphere, J. Geophys. Res. (Space Physics), doi:10.1002/jgra.50251.

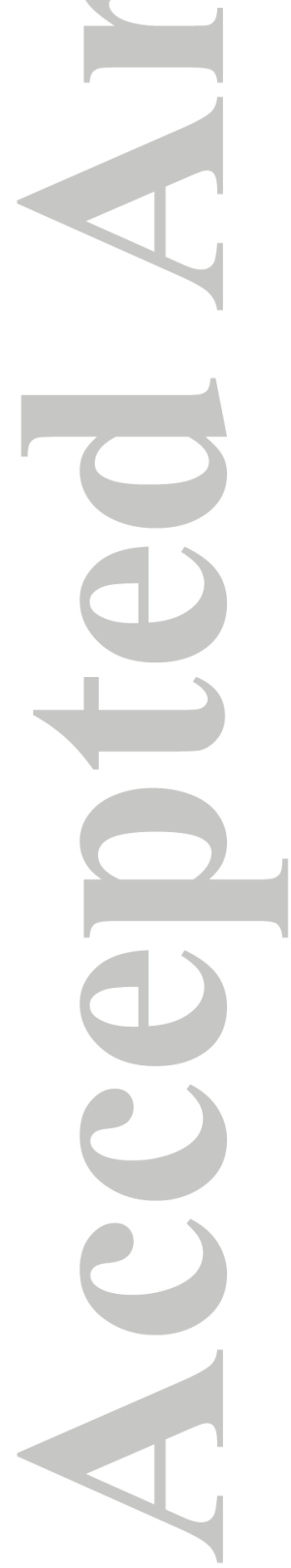

(C)2018 American Geophysical Union. All Rights Reserved. 

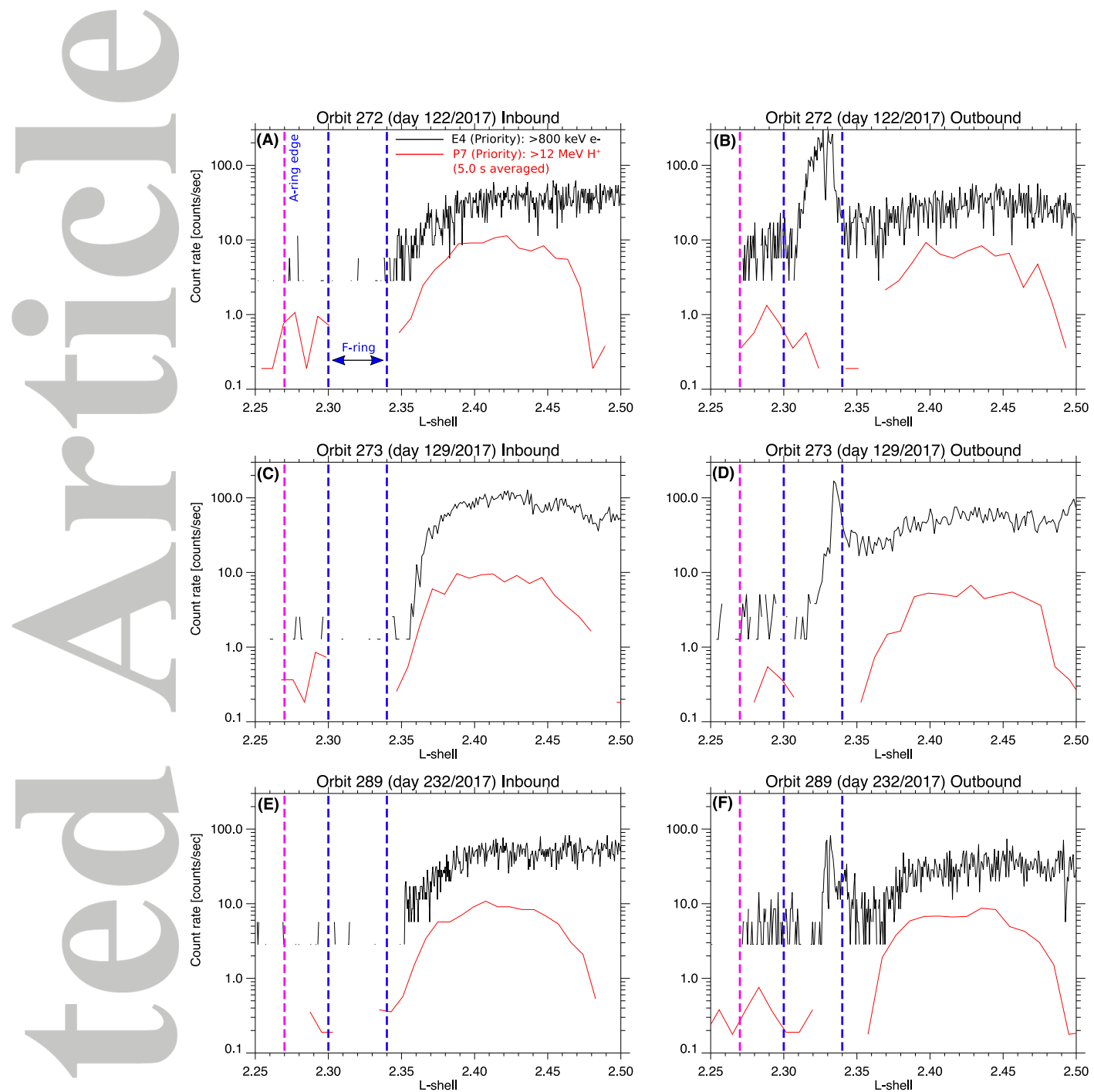

Figure 1. All panels show >800 keV electron rates (black) from LEMMS's priority channel E4 and $>12 \mathrm{MeV}$ proton rates from the priority channel P7, as a function of L-shell and for three Cassini Proximal orbits. The locations of the A and F rings are marked. The left panels are for the inbound segments, the right for the outbound.

(C)2018 American Geophysical Union. All Rights Reserved. 

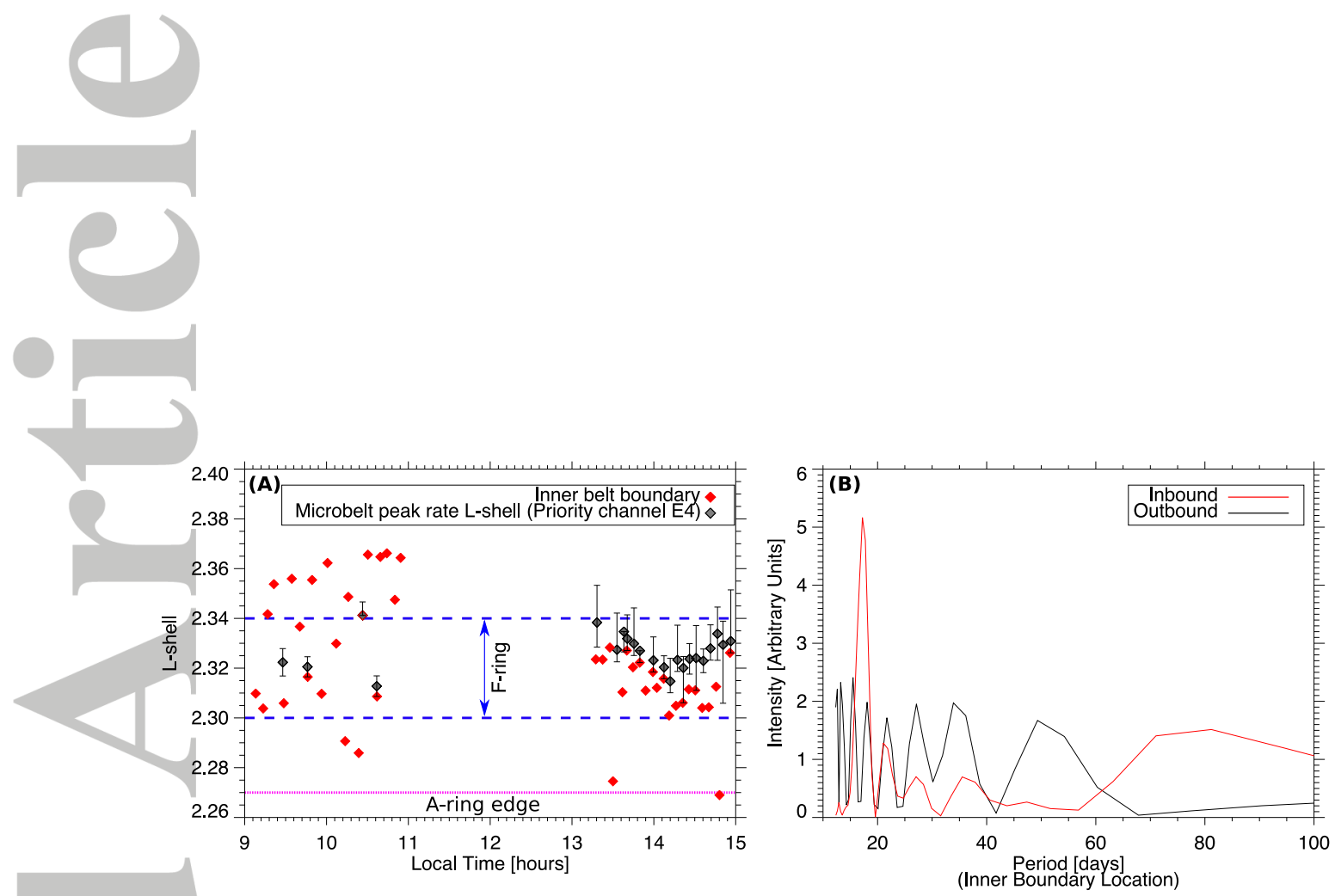

Figure 2. Left: The local time and L-shell coordinates of the inner radiation belt boundary (red diamonds) and of the microbelts (black diamonds). Each microbelt point marks the location of its peak count rate, while the error bar indicate its extent. Right: Lomb-Scargle periodograms of the $L_{b}$ time series. Red is for the inbound values (pre-noon), black for the outbound (post-noon).

(C)2018 American Geophysical Union. All Rights Reserved. 

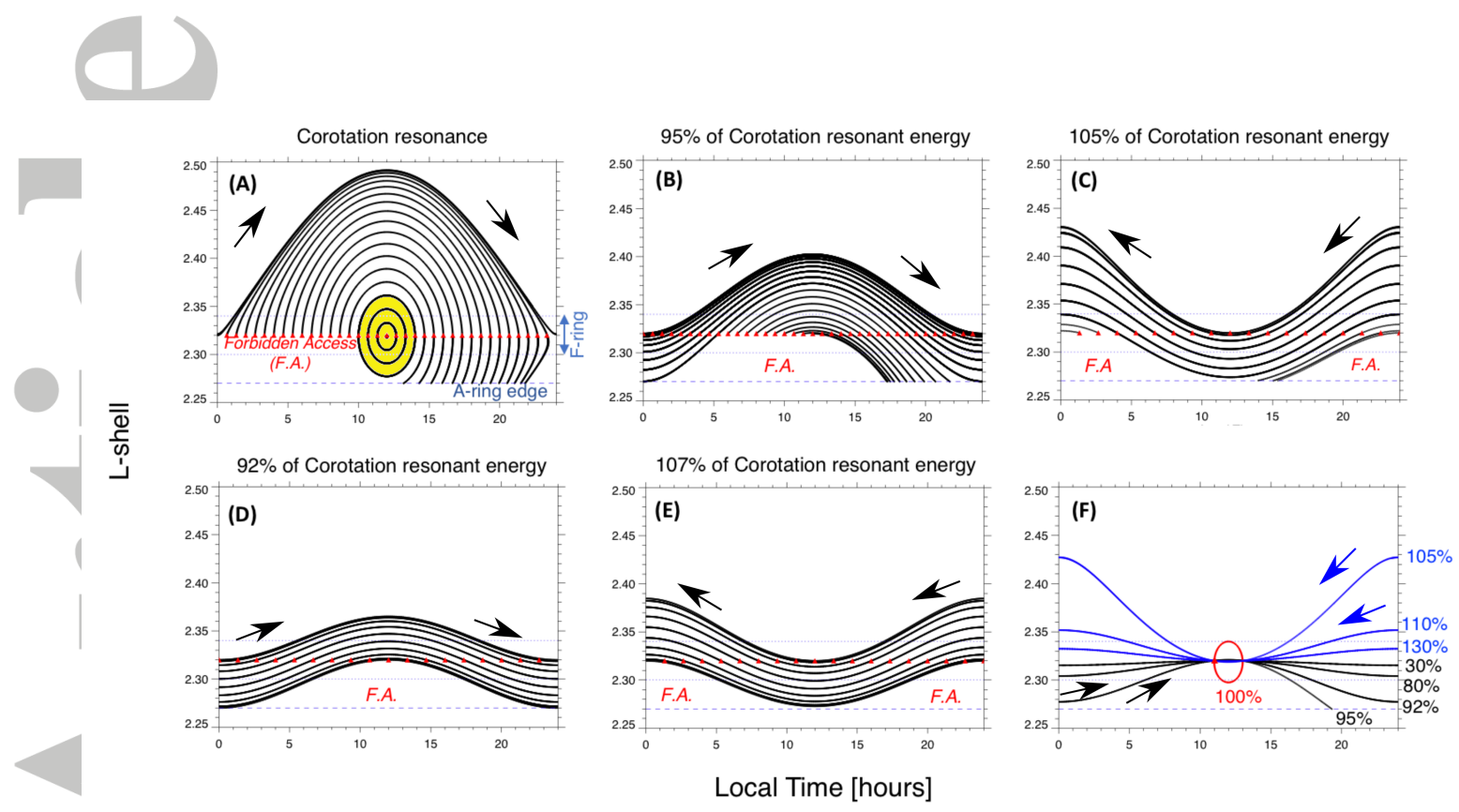

Figure 3. Drift trajectories of energetic electrons under the influence of corotation, a noon-tomidnight electric field of $0.025 \mathrm{mV} \mathrm{m}^{-1}$ and dipole magnetic drifts. Electrons reaching the A-ring are removed (non-closed trajectories). Red diamonds show the injection points and arrows the drift directions. Regions of forbidden access (F.A.) are marked in red. Panel (A) shows resonant electrons, while (B) and (C) electrons slightly above and below the resonant energy. Equatorially mirroring electrons were traced, but the picture is similar for smaller pitch angles. Panels (D) and (E) show electrons drifts not intersecting the A-ring and panel (F) the energy dispersion in L- and local time of electrons launched from 11:00. Percentages give the energy normalized to $\mathrm{E}=\mathrm{E}_{c}=3.15 \mathrm{MeV}$. Resonant electrons completed a drift orbit in $\sim 2$ weeks. For the highest energies shown here, this time is $\sim 1.5$ days and for the lowest $\sim 3$ days.

(C)2018 American Geophysical Union. All Rights Reserved. 

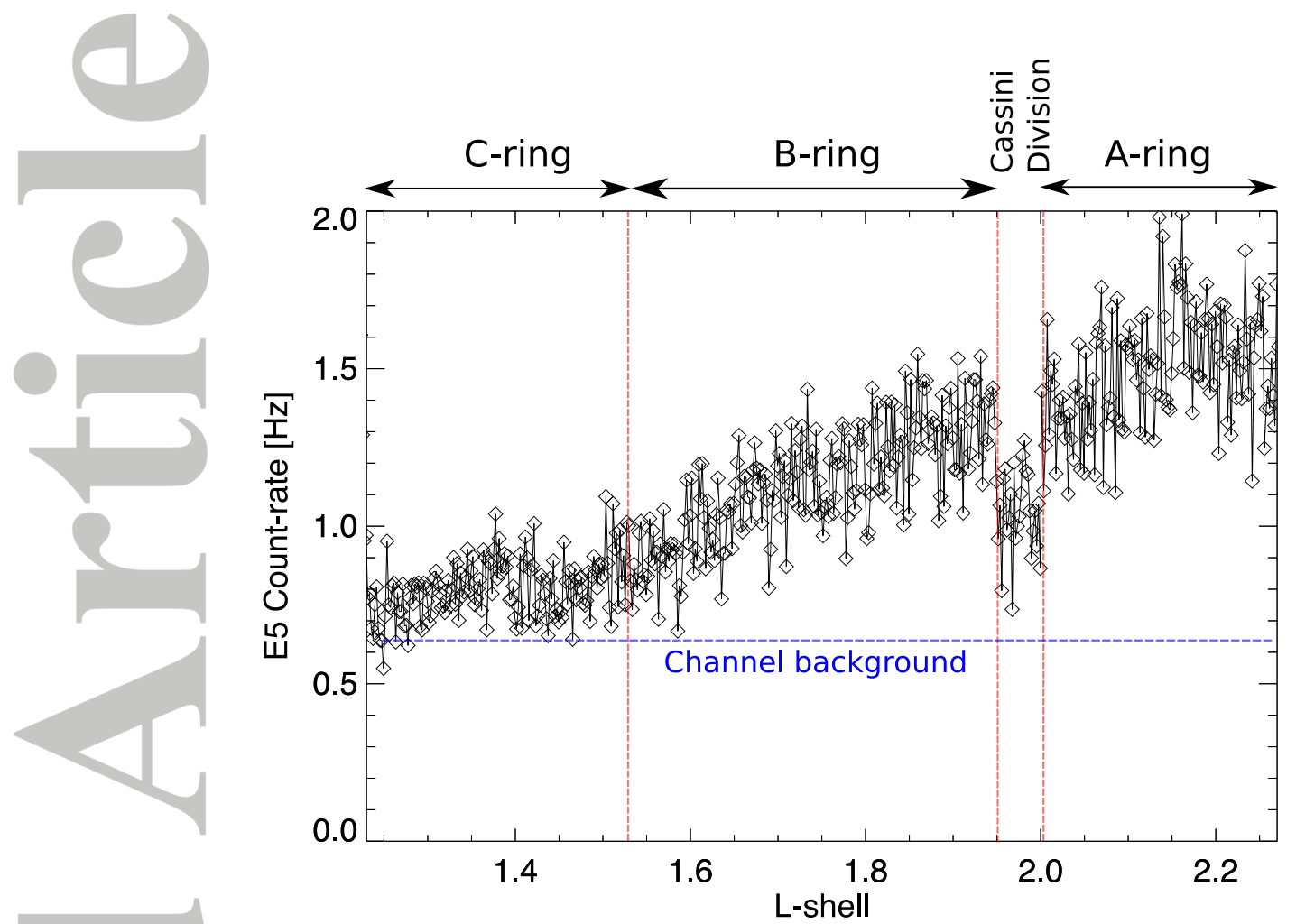

Figure 4. Proximal Orbit averaged rates of $>800 \mathrm{keV}$ electrons (channel E5 is similar to E4 but with a higher electron detection efficiency) as a function of L-shell above Saturns main rings. The E5 background level of 0.64 counts $^{-1}$ (blue line) has both a constant contribution, from Cassinis RTG Radioisotope Thermal Generators and from penetrators in the local environment (secondary gamma rays from GCR interactions with Saturns rings and atmosphere). Since gamma rays are not limited by the planets magnetic field, we measured the background using data from Cassinis final orbit and a few minutes before the end of the mission. This is a region that we do not expect any foreground signal from trapped charged particles due to the strong losses in the atmosphere.

(C)2018 American Geophysical Union. All Rights Reserved. 